\title{
The Effect of some Insecticides on Two Entomopathogenic Nematodes and Evaluation of Its Virulence on the Greater Wax Moth Galleria mellonella
}

\author{
Dina Atwa ${ }^{2}$; Mona A. Hussein ${ }^{3}$; Mostafa A. El-Khawas ${ }^{2}$; Awad A. Sarhan ${ }^{1}$; Nasser S. Mandour ${ }^{1}$ and \\ Mahmoud F. Mahmoud ${ }^{1}$ \\ ${ }^{1}$ Plant Protection Department, Faculty of Agriculture, Ismailia, 41522, Suez Canal University, Egypt. \\ ${ }^{2}$ Biological Control Department, Plant Protection Institute, Agricultural Research Center, Giza, Egypt. \\ ${ }^{3}$ Department of Pests and Plant Protection, Agricultural Division, National Research Center, Dokki, Giza, Egypt.
}

\section{Received: 3/10/2017}

\begin{abstract}
The toxicity effect of five insecticides (Dipel 2x, Radiant, Proclaim, Aphox and Coragen) on two species of entomopathogenic nematodes (EPNs) (Heterorhabditis bacteriophora BA1 and Steinernema carpocapsae BA2), were evaluated under laboratory conditions at different concentrations $100 \%, 50 \%$ and $25 \%$ of field rates (FR). Moreover, the virulence of insecticide-treated EPNs against the $1^{\text {st }}$ nymphal instar of the greater wax moth Galleria mellonella $\mathrm{L}$. (Lepidoptera: Pyralidae) was also evaluated. Data indicated that all insecticides showed negative effect on the tested infective juveniles (IJs) of EPNs and the percentages of mortality were in order of $H$. bacteriophora BA1 $>S$. carpocapsae BA2 and were concentration-dependent; significant differences existed between untreated (control) and insecticide treatments EPNs. Proclaim insecticide recorded the highest mortality rate in EPNs at all tested concentrations 24, 48 and 72 hours post treatment. Regarding the effect of tested insecticides on the virulence and the reproductive capacity of treated EPNs, data indicated that the ability of insecticide-treated EPNs to locate and infect larvae of G. mellonella decreased after exposure to insecticide and consequently reproductive capacity of EPNs was significantly reduced.
\end{abstract}

Keywords: Insecticides, Steinernema carpocapsae, Heterorhabditis bacteriophora, toxicity, Galleria mellonella, Virulence

\section{INTRODUCTION}

Generally, any integrated pest management (IPM) strategy is mainly accomplished by combining all available control methods to increase the degree of effectiveness and to obtain a long-term reliability. Entomopathogenic nematodes (EPNs) (Steinernematidae and Heterorhabditidae) and their symbiotically associated bacteria (Xenorhabdus sp. for steinernematids and Photorhabdus sp. for Heterorhabditids) are great values in the field of biological control (Kaya et al., 1998).

The infective juveniles (IJs) of EPNs usually enter their hosts via body orifices (e.g. spiracles Steinernematidae) and less often penetrate host cuticle (Heterorhabditidae). They do not kill their hosts directly; however certain mutualistic bacteria associated with EPNs release their toxins, metabolites or proteases that kill the host within 2-3 days (Poinar, 1990). EPNs are increasingly used to control a wide spectrum of insect species in different agricultural ecosystems (Kaya et al., 2006; Mahmoud, 2016).

The interaction between EPNs and other bioagents is inevitable and may determine the degree of success for these biocontrol agents. This interaction may be an antagonistic or synergistic effect (Kaya and Burlando, 1989; Mahmoud, 2007).

The objective of the current work is to evaluate the toxicity effects of five bio- and conventional insecticides at different concentrations $(100 \%, 50 \%$ and $25 \% \mathrm{FR})$ on two species of EPNs, together with evaluating its residual effects on the virulence and the reproductive capacity of the treated EPNs under laboratory conditions.

\section{MATERIALS AND METHODS}

\section{Insecticides}

The commercial products of the selected insecticides with their field rates, active ingredients, PHI (pre-harvest interval) and the target pests are listed in Table (1), Theses insecticides include two bioinsecticides (Dipel 2x and Radiant) and three conventional insecticides (proclaim, Aphox and Coragen). The tested concentrations of insecticides in the present study were $100 \%, 50 \%$ and $25 \%$ FR, which prepared one hour prior to experiments.

\section{Entomopathogenic nematodes}

The preparations of the entomopathogenic nematodes (EPNs) of the families Heterorhabditidae and Steinernematidae (Order: Rhabditida) (Heterorhabditis bacteriophora BA1 and Steinernema carpocapsae BA2)), which were used in this study, were obtained during the experiments as a suspension product from Pests and Protection Department, Mass Production Unit, National Research Center (NRC), Giza, Egypt. All tested EPNs were reared in vivo according to the technique described by Glazer and Lewis (2000). Newly emerged infective juveniles (IJs) were collected and stored firstly at $5^{\circ} \mathrm{C}$ in order to evaluate their survival. Healthy laboratory emerging infective juveniles (IJs) were collected from White traps (White, 1927) and stored in distilled water until used in the experiments.

\section{Experiments}

\section{Effect of tested insecticides on EPN (IJs)}

This experiment was conducted to evaluate the efficacy of five bio- and conventional insecticides at different rates $(25,50$ and $100 \% \mathrm{FR})$ on two species of 
EPNs. The experiment was performed at $25^{\circ} \mathrm{C}$ and data were recorded after three different intervals $(24,48$ and 72 hours). Untreated (control) experiment for the two studied EPNs species was also performed. Each treatment was replicated 4 times in a sterile tissue culture plate (24 well, $4 \mathrm{ml}$ capacity/well). Each replicate contained $1 \mathrm{ml}$ insecticide and $1 \mathrm{ml}$ EPNs IJs suspension $(2000 \mathrm{IJs} / \mathrm{ml})$, and then covered with hulls stretched plastic. Data were recorded after 24, 48 and 72 hours post treatment. Trypan blue coloring $\left(\mathrm{C}_{34} \mathrm{H}_{24} \mathrm{~N}_{6} \mathrm{Na}_{4} \mathrm{O}_{14} \mathrm{~S}_{4}\right)$ was used to discriminate between the survived and the dead nematodes. The treated infective juveniles (IJs) survived after respective tested period were kept in Wassermann test tube $(10 \mathrm{ml})$ with distilled water to evaluate their virulence.

Table (1): List of tested bio-and conventional insecticides including their active ingredients, recommended field rate (FR) and the target pests.

\begin{tabular}{|c|c|c|c|c|}
\hline Tested Insecticides & Active ingredients & FR & Target pest & PHI* (day) \\
\hline $\begin{array}{l}\text { Dipel } 2 x \\
6.4 \% \text { DF }\end{array}$ & Bacillus thuringiensis var. kurstaki & $\begin{array}{l}200 \mathrm{gm} / \text { fed } \\
150 \mathrm{gm} / \text { ton }\end{array}$ & $\begin{array}{l}\text { PTM in field } \\
\text { PTM in storage }\end{array}$ & $\mathrm{N} / \mathrm{A}^{* *}$ \\
\hline $\begin{array}{l}\text { Radiant } \\
12 \% \mathrm{SC}\end{array}$ & Spinetoram & $\begin{array}{l}120 \mathrm{~cm}^{3} / \text { fed } \\
100 \mathrm{~cm}^{3} / \text { fed }\end{array}$ & $\begin{array}{l}\text { Aphids } \\
\text { PTM }\end{array}$ & 1 \\
\hline $\begin{array}{l}\text { Proclaim oupty } \\
5 \% \text { WG }\end{array}$ & Emamectin benzoate & $60 \mathrm{gm} / \mathrm{fed}$ & PTM in field & 3 \\
\hline $\begin{array}{l}\text { Aphox } \\
50 \% \text { SC }\end{array}$ & Primicarb & $50 \mathrm{gm} / 100 \mathrm{~L}$ & Aphids & 15 \\
\hline $\begin{array}{l}\text { Coragen } \\
20 \% \mathrm{SC}\end{array}$ & Chlorantraniliprole & $60 \mathrm{~cm}^{3} / \mathrm{fed}$ & PTM in field & 7 \\
\hline
\end{tabular}

\section{Evaluation of the virulence of EPNs on full grown larvae of Galleria mellonella}

This experiment was conducted to study the impact of different concentrations of each tested insecticide on the two tested EPNs and its first generation (F1) by using the full grown larvae (FGL) of G. mellonella as laboratory host. Untreated and insecticides-treated EPNs (IJs) were taken from the previous experiment, in which the effect of the tested insecticides at 100, 50 and $25 \%$ FR were studied. In insecticide-treated EPNs, IJs were taken after 72 hours of exposure to the insecticides. Each treatment was replicated ten times. The untreated and insecticidetreated EPNs IJs were placed with the host larvae in sterile tissue culture plate ( 24 wells, $4 \mathrm{ml}$ capacity/well). 200 IJs (untreated or insecticide-treated IJs) as a suspension form was added to each larva of $G$. mellonella in each well and then covered with hulls stretched plastic. This experiment was performed under laboratory conditions of $25 \pm 2^{\circ} \mathrm{C}$ and $2: 22 \mathrm{~L} / \mathrm{D}$ photoperiod. Data were recorded for three days in terms of infected larvae with EPNs throughout the experimental period (3 days).

\section{Evaluation of the offspring of EPNs}

This experiment aimed to evaluate the offspring of insecticide-treated EPNs. The infected G. mellonella larvae (cadavers) were collected after 3 days and each cadaver was placed separately in a Petri dish in the form of White traps unit for 15 days. Then after, offspring of EPNs from the White traps unit was collected and conserved in clean Falcon tube $(50 \mathrm{ml})$ (Mahmoud and Osman, 2007). The offspring obtained from this experiment was re-examined as second generation $\left(\mathrm{F}_{2}\right)$ against FGL of $G$. mellonella, the data were recorded as mentioned above in $\mathrm{F}_{1}$ experiment.

\section{Statistical analysis}

All obtained data were analyzed using SAS package $11.0 \mathrm{v}$ (SAS Institute Inc, 2005). When F values were significant, means were separated using Fisher's least significant differences (FLSD) at a 0.05 level of significance (SAS Institute Inc, 2005). Proportional data were transformed by arcsine square root (ARC Sine) before analysis.

\section{RESULTS}

\section{Effect of tested insecticides on EPN (IJs)}

1.1. Effect of tested bio- and conventional insecticides on $\boldsymbol{H}$. bacteriophora BA1

Data showed that mortality percentage was higher in $H$. bacteriophora treated with conventional insecticides than bio-insecticides. Moreover, the 
Proclaim insecticide caused the highest mortality percentage in nematode treated with $100 \% \mathrm{FR}, 50 \% \mathrm{FR}$ and $25 \%$ FR. Data showed that the Dipel $2 x$ bioinsecticide at three different doses induced the lowest mortality percentage in treated nematodes. It was clear that the mortality percentage of $H$. bacteriophora increased significantly with increasing insecticide doses and time of exposure.

Statistical analysis showed that significant differences among treatments in percentages of mortality at $100 \%$ FR after 24 hours $(\mathrm{F}=81.84$; $\mathrm{P}$ $<0.0001), 48$ hours $(\mathrm{F}=163.42 ; \mathrm{P}<0.0001)$ and 72 hours of treatment $(\mathrm{F}=298.81 ; \mathrm{P}<0.0001$, Table 2). Pertaining to $50 \% \mathrm{FR}$, statistical analysis showed that there were significant differences among treatments in mortality percentage after 24 hours $(\mathrm{F}=46.52$; $\mathrm{P}$ $<0.0001)$, after 48 hours $(\mathrm{F}=128.91 ; \mathrm{P}<0.0001)$ and after 72 hours post treatment $(\mathrm{F}=170.39$; $\mathrm{P}<0.0001$, Table 2). As for $0.25 \% \mathrm{FR}$, it differed significantly among the tested insecticides after 24 hours $(\mathrm{F}=63.59$; $\mathrm{P}<0.0001), 48$ hours $(\mathrm{F}=182.38 ; \mathrm{P}<0.0001)$ and 72 hours post treatment $(\mathrm{F}=245.82 ; \mathrm{P}<0.0001)$.

\subsection{Effect of tested bio- and conventional insecticides on $S$. carpocapsae BA2}

The obtained data indicated that mortality percentages after 24, 48 and 72 hours in all treatments were higher than in control treatment, which gave mortality rates of $0.01,0.03$ and $0.04 \%$, respectively (Table 3).

Statistical analysis revealed that significant differences were existed among tested treatments after 24 hours $(\mathrm{F}=63.64 ; \mathrm{P}<0.0001), 48$ hours $(\mathrm{F}=64.01$; $\mathrm{P}$ $<0.0001)$ and 72 hours of treatment $(\mathrm{F}=141.58$; $\mathrm{P}$ $<0.0001$, Table 3). Apparently at $50 \%$ FR, statistical analysis indicated that significant differences were existed among treatments after 24 hours $(\mathrm{F}=65.46$; $\mathrm{P}$ $<0.0001), 48$ hours $(\mathrm{F}=100.81 ; \mathrm{P}<0.0001)$ and 48 hours of treatment $(\mathrm{F}=187.20 ; \mathrm{P}<0.0001$ Table 3$)$. As for at $25 \% \mathrm{FR}$, statistical analysis indicated that the significant differences after 24 hours $(\mathrm{F}=98.06$; $\mathrm{P}$ $=98.06), 48$ hours $(\mathrm{F}=87.02 ; \mathrm{P}<0.0001)$ and 72 hours post treatment $(\mathrm{F}=60.00 ; \mathrm{P}<0.0001)$.

\section{Evaluation of the virulence of EPNs on full grown larvae of $G$. mellonella}

\subsection{Insecticide-treated $\boldsymbol{H}$. bacteriophora BA1}

\subsubsection{Virulence of insecticide-treated $\boldsymbol{H}$. bacteriophora}

As shown in Table (4), the rate of mortality in FGL of G. mellonella, exposed to insecticide-treated IJs with $25 \%$ FR was greater than those recorded in the higher rates of insecticides (100 and 50\% FR).

As the insecticide concentration increased to $100 \% \mathrm{FR}$, there was a significant effect in the percentage of mortality $(\mathrm{F}=3.68 ; \mathrm{P}=0.0061)$ after 24 hours and $(\mathrm{F}=169.00 ; \mathrm{P}<0.0001)$ after 48 and 72 hours. As for using 50\% FR, the significant differences were $(\mathrm{F}=3.68 ; \mathrm{P}=0.0061)$ after 24 hours and $(\mathrm{F}=$ 53.35; $\mathrm{P}<0.0001)$ after 48 and 72 hours. The rate of mortality in G. mellonella FGL, exposed to insecticidetreated IJs with $25 \%$ FR recorded a significant differences and negative correlation with percentage of mortality $(\mathrm{F}=4.0 ; \mathrm{P}=0.0036)$ after 24 hours and $(\mathrm{F}=$ 27.04; $\mathrm{P}<0.0001$, Table 4 ) after 48 and 72 hours of treatment.

\subsubsection{Virulence of insecticide-treated $\boldsymbol{H}$. bacteriophora (F1)}

The mortality percentage of FGL of $G$. mellonella with $\mathrm{F}_{1}$ of treating $H$. bacteriophora at 100 , 50 and $25 \%$ FR, in control, Dipel $2 x$ and Radiant treatments were $100 \%$ after 48 and 72 hours. No significant differences were observed among the studied insecticides in terms of mortality rates in G. mellonella FGL after respective exposure periods. However at 50\% FR, significant differences were found among treatments in terms of mortality percentage after 24 hours $(\mathrm{F}=3.58 ; \mathrm{P}=0.0129) ; 48$ hours $(\mathrm{F}=39.24 ; \mathrm{P}$ $<0.0001)$ and 72 hours post treatment $(\mathrm{F}=12.80$; $\mathrm{P}$ $<0.0001$, Table 5). Obviously, at 25\% FR the significant differences among treatments after 24 hours $(\mathrm{F}=4.86$; $\mathrm{P}$ $=0.0010), 48$ hours $(\mathrm{F}=15.14 ; \mathrm{P}<0.0001)$ and 72 hours of treatment $(\mathrm{F}=5.40 ; \mathrm{P}=0.0004$, could be traced on Table 5).

\subsection{Insecticide-treated $S$. carpocapsae BA2}

\subsubsection{Virulence of insecticide-treated $S$. carpocapsae}

As shown in Table (6), the highest percentages of mortality of FGL of $G$. mellonella in the untreated (control) S. carpocapsae after 24, 48 and 72 hours post treatment were $70.00,100$ and $100 \%$, respectively. The significant differences among larval mortality percentages at $100 \% \mathrm{FR}$ after 24 hours $(\mathrm{F}=7.73$; $\mathrm{P}$ $<0.0001)$, 48 hours $(\mathrm{F}=79.20 ; \mathrm{P}<0.0001)$ and 72 hours post treatment $(\mathrm{F}=23.01 ; \mathrm{P}<0.0001)$. As for in $50 \%$ $\mathrm{FR}$, significant differences after 24 hours $(\mathrm{F}=6.95$; $\mathrm{P}<$ $0.0001), 48$ hours $(\mathrm{F}=36.00 ; \mathrm{P}<0.0001)$ and 72 hours post treatment $(\mathrm{F}=7.22 ; \mathrm{P}<0.0001$, Table 6). Apparently at $25 \% \mathrm{FR}$, there was significant differences among larval mortality percentages after 24 hours $(\mathrm{F}=$ 5.67; $\mathrm{P}=0.0003), 48$ hours $(\mathrm{F}=12.60 ; \mathrm{P}<0.0001)$ and 72 hours post treatment $(\mathrm{F}=3.26 ; \mathrm{P}=0.0121$, Table 6$)$.

\subsubsection{Virulence of insecticide-treated $S$. carpocapsae first generation (F1)}

Generally, rates of mortality in FGL of $G$. mellonella increased as the interval post exposure to the IJs increased. Also, the rates of mortality decreased as the rate of insecticides, in which the IJs were treated, increased from $25 \%$ FR to $100 \%$ FR. Moreover, statistical analysis showed no significant differences existed between treatments after 48 and 72 hours (Table 7). At 50\% FR, significant differences were found among treatments in mortality rates after 24 hours (F $=3.49 ; \mathrm{P}=0.0144), 48$ hours $(\mathrm{F}=6.75 ; \mathrm{P}=0.0002)$ and 72 hours $(\mathrm{F}=4.50 ; \mathrm{P}=0.0038$, Table 7$)$. Moreover, these differences were exited among treatments at $25 \% \mathrm{FR}$ after 24 hours $(\mathrm{F}=2.68 ; \mathrm{P}=0.0311), 48$ hours $(\mathrm{F}=3.26$; $\mathrm{P}=0.0121)$ and 72 hours of treatment $(\mathrm{F}=3.26 ; \mathrm{P}$ $=0.0120$ ). 
Table (2): Mortality percentages of $H$. bacteriophora $(2000 \mathrm{JIs} / \mathrm{ml})$ treated by different concentrations of bio- and conventional insecticides

\begin{tabular}{|c|c|c|c|c|c|c|c|c|c|}
\hline \multirow{3}{*}{ Treatment } & \multicolumn{9}{|c|}{ Mortality (\%) } \\
\hline & \multicolumn{3}{|c|}{$100 \%$ FR } & \multicolumn{3}{|c|}{$50 \%$ FR } & \multicolumn{3}{|c|}{$25 \%$ FR } \\
\hline & $24 \mathrm{~h}$ & $48 \mathrm{~h}$ & $72 \mathrm{~h}$ & $24 h$ & $48 \mathrm{~h}$ & $72 \mathrm{~h}$ & $24 h$ & $48 \mathrm{~h}$ & $72 \mathrm{~h}$ \\
\hline Control & $0.01 \mathrm{e}$ & $0.01 \mathrm{~d}$ & $0.04 \mathrm{~d}$ & $0.01 \mathrm{c}$ & $0.01 \mathrm{c}$ & $0.04 \mathrm{c}$ & $0.01 \mathrm{~d}$ & $0.01 \mathrm{~d}$ & $0.04 \mathrm{~d}$ \\
\hline Dipel 2x & $0.65 \mathrm{~d}$ & $2.15 \mathrm{c}$ & $3.84 \mathrm{c}$ & $0.53 b$ & $1.83 \mathrm{~b}$ & $3.59 \mathrm{~b}$ & $0.25 \mathrm{c}$ & $0.24 \mathrm{c}$ & $2.56 \mathrm{c}$ \\
\hline Radiant & $0.81 \mathrm{~cd}$ & $2.55 \mathrm{c}$ & $4.44 c$ & $0.80 \mathrm{~b}$ & $2.54 \mathrm{~b}$ & $4.58 \mathrm{~b}$ & $0.48 \mathrm{c}$ & $1.59 \mathrm{c}$ & $3.25 \mathrm{c}$ \\
\hline Proclaim & $1.84 \mathrm{a}$ & $8.36 \mathrm{a}$ & $19.45 \mathrm{a}$ & $1.61 \mathrm{a}$ & $6.44 \mathrm{a}$ & $12.35 \mathrm{a}$ & $2.25 \mathrm{a}$ & $6.79 a$ & $13.13 \mathrm{a}$ \\
\hline Aphox & $0.94 \mathrm{c}$ & $6.81 b$ & $15.98 \mathrm{~b}$ & $1.24 \mathrm{a}$ & $5.63 \mathrm{a}$ & $11.11 \mathrm{a}$ & $1.16 \mathrm{~b}$ & $3.68 b$ & $7.41 b$ \\
\hline Coragen & $1.41 \mathrm{~b}$ & $6.66 \mathrm{~b}$ & $17.03 \mathrm{~b}$ & $1.51 \mathrm{a}$ & $6.30 \mathrm{a}$ & $12.04 \mathrm{a}$ & $1.68 \mathrm{a}$ & $5.93 \mathrm{a}$ & $11.70 \mathrm{a}$ \\
\hline $\mathbf{F}$ & $\mathrm{F}=81.84$ & $\mathrm{~F}=163.42$ & $\mathrm{~F}=298.81$ & $\mathrm{~F}=46.52$ & $\mathrm{~F}=128.91$ & $\mathrm{~F}=170.39$ & $\mathrm{~F}=63.59$ & $\mathrm{~F}=182.38$ & $\mathrm{~F}=245.82$ \\
\hline$P$ & $\mathrm{P}<0.0001$ & $\mathrm{P}<0.0001$ & $\mathrm{P}<0.0001$ & $\mathrm{P}<0.0001$ & $\mathrm{P}<0.0001$ & $\mathrm{P}<0.0001$ & $\mathrm{P}<0.0001$ & $\mathrm{P}<0.0001$ & $\mathrm{P}<0.0001$ \\
\hline LSD & 0.85 & 1.43 & 1.72 & 1.13 & 1.46 & 1.76 & 1.15 & 1.21 & 1.46 \\
\hline
\end{tabular}

Means followed with the same letters (column wise) are not significantly different.

Obtained values of $\%$ mortality were transformed to ARC Sine before conducting ANOVA.

$\mathrm{FR}=$ field rate of insecticides.

Number of replicates for each treatment (4)

Table (3): Mortality percentages of S. carpocapsae (2000JIs $/ \mathrm{ml}$ ) treated by different concentrations of the tested bio- and conventional insecticides

\begin{tabular}{|c|c|c|c|c|c|c|c|c|c|c|}
\hline \multirow{3}{*}{ Treatment } & \multicolumn{10}{|c|}{ \% Mortality (Mean) } \\
\hline & \multicolumn{3}{|c|}{$100 \%$ FR } & \multicolumn{2}{|c|}{$50 \%$ FR } & \multirow[b]{2}{*}{$72 \mathrm{~h}$} & \multicolumn{4}{|c|}{$25 \%$ FR } \\
\hline & $24 \mathrm{~h}$ & $48 \mathrm{~h}$ & $72 \mathrm{~h}$ & $24 \mathrm{~h}$ & $48 \mathrm{~h}$ & & $24 h$ & $48 \mathrm{~h}$ & 72 & \\
\hline Control & $0.01 \mathrm{c}$ & $0.03 c$ & $0.04 d$ & $0.01 \mathrm{c}$ & $0.03 c$ & $0.04 \mathrm{c}$ & $0.01 d$ & $0.03 \mathrm{e}$ & 0. & \\
\hline Dipel $2 x$ & $0.49 b$ & $1.11 \mathrm{~b}$ & $2.36 \mathrm{c}$ & $0.30 \mathrm{~b}$ & $1.08 \mathrm{~b}$ & $2.24 b$ & $0.05 \mathrm{~d}$ & $0.38 \mathrm{~d}$ & 0.8 & \\
\hline Radiant & $0.54 b$ & $1.33 \mathrm{~b}$ & $2.60 \mathrm{c}$ & $0.48 b$ & $1.43 b$ & $2.85 b$ & $0.24 \mathrm{c}$ & $0.94 \mathrm{c}$ & 2.1 & \\
\hline Proclaim & $1.49 \mathrm{a}$ & $4.79 \mathrm{a}$ & $11.53 \mathrm{a}$ & $1.68 \mathrm{a}$ & $4.65 \mathrm{a}$ & $8.75 a$ & $1.45 \mathrm{a}$ & $4.01 \mathrm{a}$ & 7.6 & \\
\hline Aphox & $1.16 \mathrm{a}$ & $3.99 \mathrm{a}$ & $8.83 b$ & $1.45 \mathrm{a}$ & $4.20 \mathrm{a}$ & $7.75 \mathrm{a}$ & $1.01 \mathrm{~b}$ & $2.66 b$ & & \\
\hline Coragen & $1.30 \mathrm{a}$ & $4.28 \mathrm{a}$ & $10.06 \mathrm{ab}$ & $1.54 \mathrm{a}$ & $4.43 \mathrm{a}$ & $8.41 \mathrm{a}$ & $1.24 \mathrm{ab}$ & $3.69 \mathrm{a}$ & & \\
\hline $\mathbf{F}$ & $\mathrm{F}=63.64$ & $\mathrm{~F}=64.01$ & $\mathrm{~F}=141.58$ & $\mathrm{~F}=65.46$ & $\mathrm{~F}=100.81$ & $\mathrm{~F}=187.20$ & $\mathrm{~F}=98.06$ & $\mathrm{~F}=87.02$ & & 00 \\
\hline$P$ & $\mathrm{P}<0.0001$ & $\mathrm{P}<0.0001$ & $\mathrm{P}<0.0001$ & $\mathrm{P}<0.0001$ & $\mathrm{P}<0.0001$ & $\mathrm{P}<0.0001$ & $\mathrm{P}=98.0$ & & $\mathrm{P}<0.0001$ & $\mathrm{P}<0.0001$ \\
\hline LSD & 0.93 & 1.73 & 1.85 & 1.04 & 1.39 & 1.40 & 0.85 & & 1.40 & 2.33 \\
\hline
\end{tabular}

Means followed with the same letters (column wise) are not significantly different.

Obtained values of $\%$ mortality were transformed to ARC Sine before conducting ANOVA.

*number of replicate for each treatment.

$\mathrm{FR}=$ field rate of insecticides.

Number of replicates for each treatment (4). 
Table (4): Mortality percentages of FGL of G. mellonella exposed to insecticide-treated $H$. bacteriophora at different concentrations

\begin{tabular}{|c|c|c|c|c|c|c|c|c|c|}
\hline \multirow{3}{*}{ Treatment } & \multicolumn{9}{|c|}{ \% Mortality (Mean) } \\
\hline & \multicolumn{3}{|c|}{$100 \%$ FR } & \multicolumn{3}{|c|}{$50 \%$ FR } & \multicolumn{3}{|c|}{$25 \%$ FR } \\
\hline & $24 \mathrm{~h}$ & $48 \mathrm{~h}$ & $72 \mathrm{~h}$ & $24 h$ & $48 \mathrm{~h}$ & $72 \mathrm{~h}$ & $24 h$ & $48 \mathrm{~h}$ & $72 \mathrm{~h}$ \\
\hline Control & $50.00 \mathrm{a}$ & $100 \mathrm{a}$ & $100 \mathrm{a}$ & $50.00 \mathrm{a}$ & $100 \mathrm{a}$ & $100 \mathrm{a}$ & $50.00 \mathrm{a}$ & $100 \mathrm{a}$ & $100 \mathrm{a}$ \\
\hline Dipel 2x & $30.00 \mathrm{ab}$ & $100 \mathrm{a}$ & $100 \mathrm{a}$ & $30.00 \mathrm{ab}$ & $100 \mathrm{a}$ & $100 \mathrm{a}$ & $40.00 \mathrm{a}$ & $100 \mathrm{a}$ & $100 \mathrm{a}$ \\
\hline Radiant & $30.00 \mathrm{ab}$ & $100 \mathrm{a}$ & $100 \mathrm{a}$ & $30.00 \mathrm{ab}$ & $100 \mathrm{a}$ & $100 \mathrm{a}$ & $30.00 \mathrm{ab}$ & $100 \mathrm{a}$ & $100 \mathrm{a}$ \\
\hline Proclaim & $0 \mathrm{~b}$ & $0 \mathrm{~b}$ & $0 \mathrm{~b}$ & $0 \mathrm{~b}$ & $0 \mathrm{c}$ & $0 \mathrm{c}$ & $0 \mathrm{~b}$ & $10.00 \mathrm{~b}$ & $10.00 \mathrm{~b}$ \\
\hline Aphox & $0 \mathrm{~b}$ & $10.00 \mathrm{~b}$ & $10.00 \mathrm{~b}$ & $0 \mathrm{~b}$ & $20.00 \mathrm{~b}$ & $20.00 \mathrm{~b}$ & $0 \mathrm{~b}$ & $20.00 \mathrm{~b}$ & $20.00 \mathrm{~b}$ \\
\hline Coragen & $0 \mathrm{~b}$ & $0 \mathrm{~b}$ & $0 \mathrm{~b}$ & $0 \mathrm{~b}$ & $10.00 \mathrm{bc}$ & $10.00 \mathrm{bc}$ & $0 \mathrm{~b}$ & $20.00 \mathrm{~b}$ & $20.00 \mathrm{~b}$ \\
\hline $\begin{array}{l}\mathbf{F} \\
P\end{array}$ & $\begin{array}{l}F=3.68 \\
P=0.0061\end{array}$ & $\begin{array}{l}\mathrm{F}=169.00 \\
\mathrm{P}<0.0001\end{array}$ & $\begin{array}{l}\mathrm{F}=169.00 \\
\mathrm{P}<0.0001\end{array}$ & $\begin{array}{l}F=3.68 \\
P=0.0061\end{array}$ & $\begin{array}{l}\mathrm{F}=53.35 \\
\mathrm{P}<0.0001\end{array}$ & $\begin{array}{l}\mathrm{F}=53.35 \\
\mathrm{P}<0.0001\end{array}$ & $\begin{array}{l}\mathrm{F}=4.01 ; \\
\mathrm{P}=0.0036\end{array}$ & $\begin{array}{l}\mathrm{F}=27.04 \\
\mathrm{P}<0.0001\end{array}$ & $\begin{array}{l}\mathrm{F}=27.04 ; \\
\mathrm{P}<0.0001\end{array}$ \\
\hline LSD & 28.42 & 10.42 & 10.42 & 28.42 & 17.36 & 17.36 & 29.05 & 22.24 & 22.24 \\
\hline
\end{tabular}

Means followed with the same letters (column wise) are not significantly different.

Obtained values of \% mortality were transformed to ARC Sine before conducting ANOVA.

Number of replicates for each treatment (10)

$\mathrm{FR}=$ field rate of insecticides.

$\mathrm{FGL}=$ full grown larvae of $G$. mellonella

Table (5): Mortality percentages of FGL of G. mellonella exposed to $\mathrm{F}_{1}$ of insecticide-treated H. bacteriophora at different concentrations

\begin{tabular}{|c|c|c|c|c|c|c|c|c|c|}
\hline \multirow{3}{*}{ Treatment } & \multicolumn{9}{|c|}{ \% Mortality (Mean) } \\
\hline & \multicolumn{3}{|c|}{$100 \%$ FR } & \multicolumn{3}{|c|}{$50 \%$ FR } & \multicolumn{3}{|c|}{$25 \%$ FR } \\
\hline & $24 \mathrm{~h}$ & $48 \mathrm{~h}$ & $72 \mathrm{~h}$ & $24 \mathrm{~h}$ & $48 \mathrm{~h}$ & $72 \mathrm{~h}$ & $24 h$ & $48 \mathrm{~h}$ & $72 \mathrm{~h}$ \\
\hline Control & $50.00 \mathrm{a}$ & $100 \mathrm{a}$ & $100 \mathrm{a}$ & $50.00 \mathrm{a}$ & $100 \mathrm{a}$ & $100 \mathrm{a}$ & $50.00 \mathrm{a}$ & $100 \mathrm{a}$ & $100 \mathrm{a}$ \\
\hline Dipel 2x & $40.00 \mathrm{a}$ & $100 \mathrm{a}$ & $100 \mathrm{a}$ & $40.00 \mathrm{a}$ & $100 \mathrm{a}$ & $100 \mathrm{a}$ & $40.00 \mathrm{a}$ & $100 \mathrm{a}$ & $100 \mathrm{a}$ \\
\hline Radiant & $40.00 \mathrm{a}$ & $100 \mathrm{a}$ & $100 \mathrm{a}$ & $40.00 \mathrm{a}$ & $100 \mathrm{a}$ & $100 \mathrm{a}$ & $50.00 \mathrm{a}$ & $100 \mathrm{a}$ & $100 \mathrm{a}$ \\
\hline Proclaim & - & - & - & - & - & - & $\mathrm{Ob}$ & $30.00 \mathrm{~b}$ & $50.00 \mathrm{~b}$ \\
\hline Aphox & - & - & - & $0 \mathrm{~b}$ & $20.00 \mathrm{~b}$ & $40.00 \mathrm{~b}$ & $0 \mathrm{~b}$ & $20.00 \mathrm{~b}$ & $50.00 \mathrm{~b}$ \\
\hline Coragen & - & - & - & $0 \mathrm{~b}$ & $10.00 \mathrm{~b}$ & $30.00 \mathrm{~b}$ & $0 \mathrm{~b}$ & $30.00 \mathrm{~b}$ & $50.00 \mathrm{~b}$ \\
\hline $\begin{array}{l}\text { F } \\
P\end{array}$ & $\begin{array}{l}\mathrm{F}=0.12 ; \\
\mathrm{P}=0.8845\end{array}$ & $\begin{array}{l}\mathrm{F}=0 \\
\mathrm{P}=0\end{array}$ & $\begin{array}{l}\mathrm{F}=0 \\
\mathrm{P}=0\end{array}$ & $\begin{array}{l}\mathrm{F}=3.58 \\
\mathrm{P}=0.0129\end{array}$ & $\begin{array}{l}\mathrm{F}=39.24 \\
\mathrm{P}<0.0001\end{array}$ & $\begin{array}{l}\mathrm{F}=12.80 \\
\mathrm{P}<0.0001\end{array}$ & $\begin{array}{l}F=4.86 \\
P=0.0010\end{array}$ & $\mathrm{~F}=15.14 ; \mathrm{P}<0.0001$ & $\begin{array}{l}\mathrm{F}=5.40 \\
\mathrm{P}=0.0004\end{array}$ \\
\hline LSD & 42.94 & - & - & 32.65 & 19.11 & 25.64 & 29.87 & 26.45 & 30.07 \\
\hline
\end{tabular}

Means followed with the same letters (column wise) are not significantly different.

Obtained values of $\%$ mortality were transformed to ARC Sine before conducting ANOVA.

Number of replicates for each treatment (10).

- The treatments were excluded during statistical analysis.

$\mathrm{FR}=$ field rate of insecticides.

$\mathrm{FGL}=$ full grown larvae of $G$. mellonella 
Table (6): Mortality percentages of FGL of G. mellonella exposed to insecticide-treated S. carpocapsae at different concentrations

\begin{tabular}{|c|c|c|c|c|c|c|c|c|c|}
\hline \multirow{3}{*}{ Treatment } & \multicolumn{9}{|c|}{ \% Mortality (Mean) } \\
\hline & \multicolumn{3}{|c|}{$100 \%$ FR } & \multicolumn{3}{|c|}{$50 \%$ FR } & \multicolumn{3}{|c|}{$25 \%$ FR } \\
\hline & $24 h$ & $48 h$ & $72 h$ & $24 h$ & $48 \mathrm{~h}$ & $72 h$ & $24 h$ & $48 h$ & $72 h$ \\
\hline Control & $70.00 \mathrm{a}$ & $100 \mathrm{a}$ & $100 \mathrm{a}$ & $70.00 \mathrm{a}$ & $100 \mathrm{a}$ & $100 \mathrm{a}$ & $70.00 \mathrm{a}$ & $100 \mathrm{a}$ & $100 \mathrm{a}$ \\
\hline Dipel 2x & $50.00 \mathrm{a}$ & $100 \mathrm{a}$ & $100 \mathrm{a}$ & $50.00 \mathrm{a}$ & $100 \mathrm{a}$ & $100 \mathrm{a}$ & $70.00 \mathrm{a}$ & $100 \mathrm{a}$ & $100 \mathrm{a}$ \\
\hline Radiant & $50.00 \mathrm{a}$ & $100 \mathrm{a}$ & $100 \mathrm{a}$ & $60.00 \mathrm{a}$ & $100 \mathrm{a}$ & $100 \mathrm{a}$ & $60.00 \mathrm{a}$ & $100 \mathrm{a}$ & $100 \mathrm{a}$ \\
\hline Proclaim & $0 \mathrm{~b}$ & $0 \mathrm{~b}$ & $10.00 \mathrm{~b}$ & $10.00 \mathrm{~b}$ & $20.00 \mathrm{~b}$ & $40.00 \mathrm{~b}$ & $10.00 \mathrm{~b}$ & $30.00 \mathrm{~b}$ & $60.00 \mathrm{~b}$ \\
\hline Aphox & $0 \mathrm{~b}$ & $10.00 \mathrm{~b}$ & $30.00 \mathrm{~b}$ & $0 \mathrm{~b}$ & $10.00 \mathrm{~b}$ & $40.00 \mathrm{~b}$ & $10.00 \mathrm{~b}$ & $30.00 \mathrm{~b}$ & $70.00 \mathrm{ab}$ \\
\hline Coragen & $0 \mathrm{~b}$ & $0 \mathrm{~b}$ & $20.00 \mathrm{~b}$ & $0 \mathrm{~b}$ & $10.00 \mathrm{~b}$ & $50.00 \mathrm{~b}$ & $10.00 \mathrm{~b}$ & $30.00 \mathrm{~b}$ & $60.00 \mathrm{~b}$ \\
\hline $\mathbf{F}$ & $F=7.73$ & $\mathrm{~F}=79.20$ & $\mathrm{~F}=23.01$ & $F=6.95$ & $\mathrm{~F}=36.00$ & $F=7.22$ & $\mathrm{~F}=5.67$ & $\mathrm{~F}=12.60$ & $\mathrm{~F}=3.26$ \\
\hline$P$ & $\mathrm{P}<0.0001$ & $\mathrm{P}<0.0001$ & $\mathrm{P}<0.0001$ & $\mathrm{P}<0.0001$ & $\mathrm{P}<0.0001$ & $\mathrm{P}<0.0001$ & $\mathrm{P}=0.0003$ & $\mathrm{P}<0.0001$ & $\mathrm{P}=0.0121$ \\
\hline LSD & 29.26 & 14.73 & 23.55 & 30.87 & 20.25 & 29.67 & 33.49 & 27.56 & 28.85 \\
\hline
\end{tabular}

Means followed with the same letters (column wise) are not significantly different.

Obtained values of $\%$ mortality were transformed to ARC Sine before conducting ANOVA.

Number of replicates for each treatment (10).

$\mathrm{FR}=$ field rate of insecticides.

$\mathrm{FGL}=$ full grown larvae of $G$. mellonella.

Table (7): Mortality percentages of FGL of G. mellonella exposed to $\mathrm{F}_{1}$ of insecticide-treated $S$. carpocapsae at different concentrations

\begin{tabular}{|c|c|c|c|c|c|c|c|c|c|}
\hline \multirow{3}{*}{ Treatment } & \multicolumn{9}{|c|}{ \% Mortality (Mean) } \\
\hline & \multicolumn{3}{|c|}{$100 \%$ FR } & \multicolumn{3}{|c|}{$50 \%$ FR } & \multicolumn{3}{|c|}{$25 \%$ FR } \\
\hline & $24 h$ & $48 \mathrm{~h}$ & $72 \mathrm{~h}$ & $24 h$ & $48 \mathrm{~h}$ & $72 \mathrm{~h}$ & $24 \mathrm{~h}$ & $48 \mathrm{~h}$ & $72 \mathrm{~h}$ \\
\hline Control & $70.00 \mathrm{a}$ & $100 \mathrm{a}$ & $100 \mathrm{a}$ & $70.00 \mathrm{a}$ & $100 \mathrm{a}$ & $100 \mathrm{a}$ & $70.00 \mathrm{a}$ & $100 \mathrm{a}$ & $100 \mathrm{a}$ \\
\hline Dipel 2x & $60.00 \mathrm{a}$ & $100 \mathrm{a}$ & $100 \mathrm{a}$ & $60.00 \mathrm{ab}$ & $100 \mathrm{a}$ & $100 \mathrm{a}$ & $60.00 \mathrm{ab}$ & $100 \mathrm{a}$ & $100 \mathrm{a}$ \\
\hline Radiant & $60.00 \mathrm{a}$ & $100 \mathrm{a}$ & $100 \mathrm{a}$ & $60.00 \mathrm{ab}$ & $100 \mathrm{a}$ & $100 \mathrm{a}$ & $70.00 \mathrm{a}$ & $100 \mathrm{a}$ & $100 \mathrm{a}$ \\
\hline Proclaim & - & - & - & - & - & - & $20.00 \mathrm{~b}$ & $60.00 \mathrm{~b}$ & $60.00 \mathrm{~b}$ \\
\hline Aphox & - & - & - & $10.00 \mathrm{c}$ & $50.00 \mathrm{~b}$ & $60.00 \mathrm{~b}$ & $30.00 \mathrm{ab}$ & $70.00 \mathrm{ab}$ & $80.00 \mathrm{ab}$ \\
\hline Coragen & - & - & - & $20.00 \mathrm{bc}$ & $50.00 \mathrm{~b}$ & $60.00 \mathrm{~b}$ & $20.00 \mathrm{~b}$ & $60.00 \mathrm{~b}$ & $60.00 \mathrm{~b}$ \\
\hline $\begin{array}{l}\mathbf{F} \\
P\end{array}$ & $\begin{array}{l}\mathrm{F}=0.13 ; \\
\mathrm{P}=0.8783\end{array}$ & $\begin{array}{l}\mathrm{F}=0 \\
\mathrm{P}=0\end{array}$ & $\begin{array}{l}\mathrm{F}=0 \\
\mathrm{P}=0\end{array}$ & $\begin{array}{l}\mathrm{F}=3.49 \\
\mathrm{P}=0.0144\end{array}$ & $\begin{array}{l}\mathrm{F}=6.75 \\
\mathrm{P}=0.0002\end{array}$ & $\begin{array}{l}\mathrm{F}=4.50 \\
\mathrm{P}=0.0038\end{array}$ & $\begin{array}{l}F=2.68 \\
P=0.0311\end{array}$ & $\begin{array}{l}\mathrm{F}=3.26 \\
\mathrm{P}=0.0121\end{array}$ & $\begin{array}{l}F=3.26 \\
P=0.0120\end{array}$ \\
\hline LSD & 41.75 & - & - & 37.05 & 27.02 & 26.48 & 37.88 & 28.85 & 27.78 \\
\hline
\end{tabular}

Means followed with the same letters (column wise) are not significantly different.

Obtained values of $\%$ mortality were transformed to ARC Sine before conducting ANOVA.

Obtained values of $\%$ mortality were transfor
Number of replicates for each treatment (10).

Number of replicates for each treatment $(10)$.
- The treatments were excluded during statistical analysis

$\mathrm{FR}=$ field rate

$\mathrm{FGL}=$ full grown larvae of $\mathrm{G}$. mellonella 


\section{Evaluation of the offspring of EPNs}

\subsection{Insecticide-treated $H$. bacteriophora BA1}

\subsubsection{First generation offspring of the insecticide- treated $\boldsymbol{H}$. bacteriophora}

The bioinsecticides potency of Emamectin benzoate represented as Proclaim had the least $F_{1}$ offspring of $H$. bacteriophora IJs in FGL of $G$. mellonella cadavers, and there were significant differences between the tested bio- and conventional insecticides at $100 \% \mathrm{FR}(\mathrm{F}=266.72 ; \mathrm{P}<0.0001), 50 \%$ $\mathrm{FR}(\mathrm{F}=227.81 ; \mathrm{P}<0.0001)$ and $25 \% \mathrm{FR}(\mathrm{F}=285.42 ; \mathrm{P}$ $<0.0001$, Fig 1a), post treatment.

\subsubsection{Second generation offspring of the insecticide- treated $\boldsymbol{H}$. bacteriophora}

Generally, the $\mathrm{F}_{2}$ IJs offspring of treated $H$. bacteriophora increased slightly with decreasing the rate of application of each tested insecticides. The obtained results indicated that all treatments had a profound significant effect on the $\mathrm{F}_{2}$ offspring of the insecticide-treated $H$. bacteriophora at $100 \% \mathrm{FR}(\mathrm{F}=$ 4755.68; $\mathrm{P}<0.0001), 50 \% \mathrm{FR}(\mathrm{F}=6672.72$; $\mathrm{P}<0.0001)$ and $50 \% \mathrm{FR}(\mathrm{F}=8021.37$; $\mathrm{P}<0.0001$, Fig $1 \mathrm{~b})$, after 15 days post treatment.

\subsection{Insecticide-treated $S$. carpocapsae $\mathrm{BA2}$}

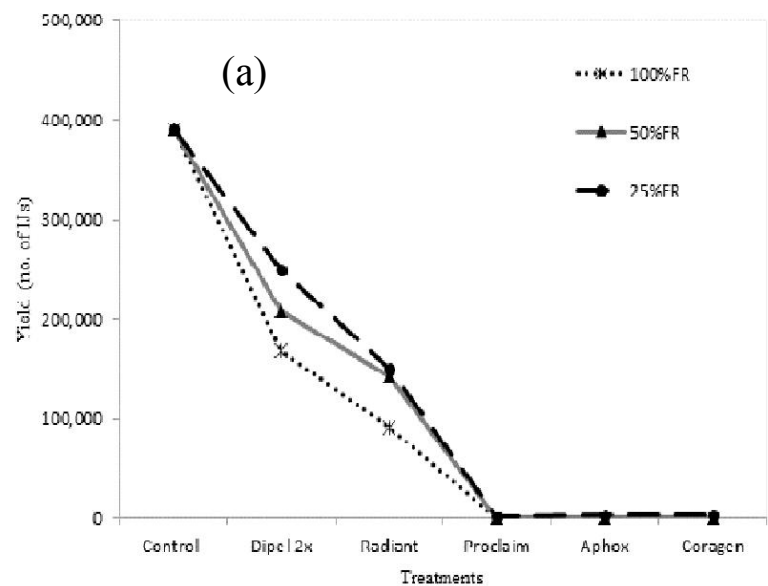

\subsubsection{First generation offspring of the insecticide- treated $S$. carpocapsae}

Pertaining tothe $\mathrm{F}_{1}$ offspringof the insecticidetreated $S$. carpocapsae produced from FGL of $G$. mellonella cadavers in all tested concentrations was in the order of control $>$ Dipel $2 x>$ Radiant $>$ Aphox $>$ Coragen $>$ Proclaim (Fig 2a). Significant differences were existed among the tested bio- and conventional insecticides after 15 days post treatment at $100 \%$ FR $(\mathrm{F}=400.22 ; \mathrm{P}<0.0001), 50 \% \mathrm{FR} \quad(\mathrm{F}=444.18 ; \mathrm{P}$ $<0.0001)$ and $25 \% \mathrm{FR}(\mathrm{F}=407.15 ; \mathrm{P}<0.0001)$. No significant differences were detected among Proclaim, Aphox and Coragen treatments (Fig 2a).

\subsubsection{Second generation offspring of the insecticide- treated $S$. carpocapsae}

The $\mathrm{F}_{2}$ of the treated $S$. carpocapsae showed the greatest offspring rate in untreated (control), followed by Dipel $2 \mathrm{x}$ and Radiant treatments at all tested concentrations. Statistical analysis revealed that the tested insecticides caused significant differences in the $\mathrm{F}_{2}$ offspringof insecticide-treated $S$. carpocapsae at $100 \%$ FR $(\mathrm{F}=51134.0 ; \mathrm{P}<0.0001), 50 \%$ FR $(\mathrm{F}=$ 107028; $\mathrm{P}<0.0001)$ and $25 \% \mathrm{FR}(\mathrm{F}=144675 ; \mathrm{P}$ $<0.0001$ ) after 15 days post treatment (Fig 2b).

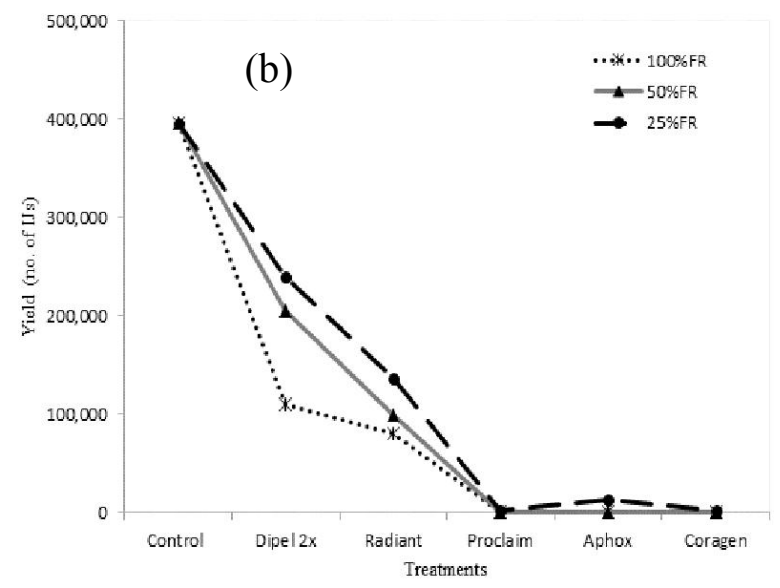

Fig (1): Number of $\mathrm{F}_{1}(\mathrm{a})$ and $\mathrm{F}_{2}$ (b) of the insecticide-treated $H$. bacteriophora (IJs) from FGL of G. mellonella cadavers after 15 days post treatment
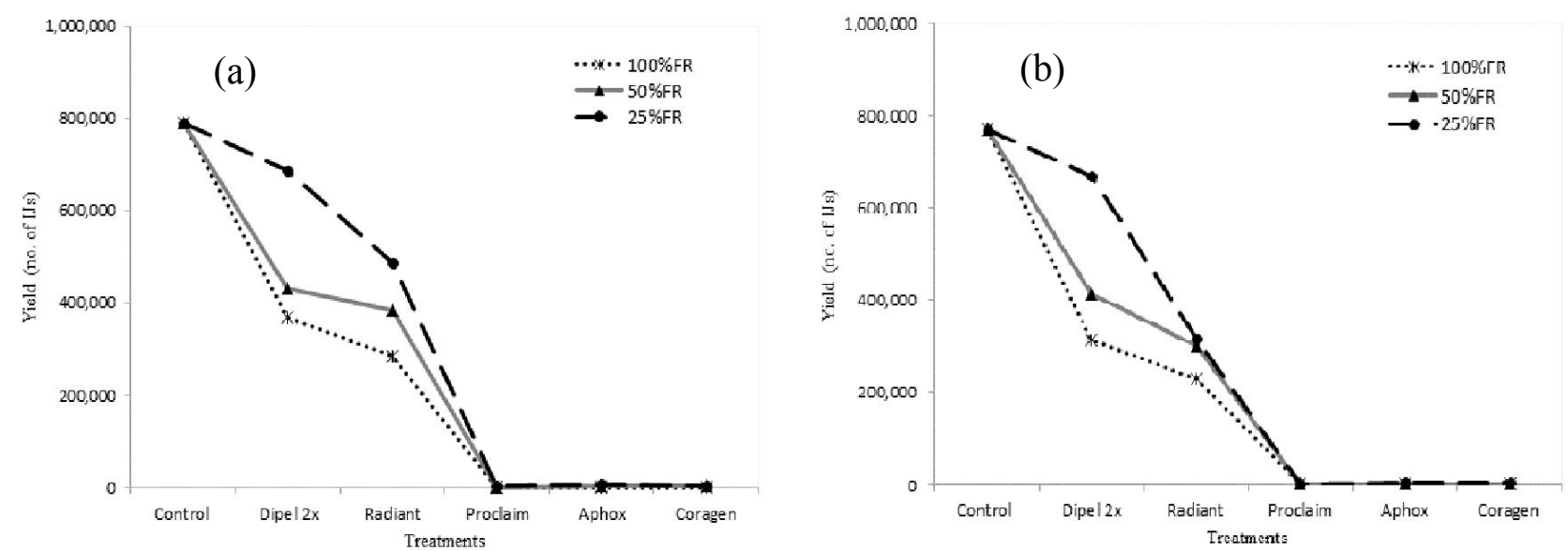

Fig (2): Number of $\mathrm{F}_{1}(\mathrm{a})$ and $\mathrm{F}_{2}$ (b) of the insecticide-treated $S$. carpocapsae (IJs) from FGL of G. mellonella cadavers after 15 days post treatment. 


\section{DISCUSSION}

The present study evaluated the effect of five bioand conventional insecticides at three different rates on two species of EPNs (IJs). Fortunately, the tested EPN (IJs) were rather compatible with tested bio- and conventional insecticides. Similar results were also reported by Laznik and Trdan (2013), who found that Steinernema sp. (IJs) was the most tolerant EPN to insecticides at all tested concentrations. Mahmoud et al. (2016) stated that combination between EPNs and insecticides such as Azadirachtin and Neonicotinoid increased the efficacy of EPNs toward host pests. Also, Bortoluzzi et al. (2013) found that insecticides had no effect on the symbiotic bacteria of EPNs, but they were harmful to EPNs infectivity. In addition, there is a unique phenomenon of "stretching" was observed in the survived insecticide-treated EPN (IJs) particularly at the highest rate 3 days post treatment. With respect to Dipel $2 \mathrm{x}^{\circledR}$ as a $B$. thuringiensis product, and Radiant ${ }^{\circledR}$ insecticide as a Spinetoram product, they were slightly toxic to the tested EPNs. This conclusion agreed to some extent with those obtained by Morales-Rodriguez and Peck (2009) who mentioned that the synergism integration between EPN (IJs) and Dipel $2 \mathrm{x}^{\circledR}$ yielded promising alternatives to control the host insects, which one of its life stages in the soil. However, this study revealed the effect on the reproductive capacity of treated EPNs female with previous insecticides as compared to untreated EPNs, especially on the EPNs first generation performance, that obtained from insecticide-treated EPNs, under the same conditions. The combination within EPNs and Dipel $2 x^{\circledR}$ or Radiant $^{\circledR}$ insecticides might be successful to control insect pests, as long as insecticide concentration is low. Primicarb (Aphox ${ }^{\circledR}$ ) lead to reduction in the survival and infectivity of tested EPNs, but less than Proclaim ${ }^{\circledR}$ insecticide. It is likely that the effect on the mortality rate and biological properties could be happened at the long-term of application.

This study evaluated also the virulence of survived IJs obtained from insecticide treatment by using G. mellonella as insect host under laboratory conditions. Insecticide-treated EPN (IJs) at highest concentration $(100 \%$ FR) showed no significant effect on the mortality of tested insect pests compared to insecticide-treated EPN (IJs) at lowest concentration (25\% FR) or untreated control EPN (IJs). The present findings are consistent with those of Sznyk-Basalyga et al. (2002) and Sznyk-Basalyga and Bednarek (2003) who realized that the rise of insecticide concentrations led to decrease in EPNs activity. In addition, the results indicated that $H$. bacteriophora and $S$. carpocapsae had a high degree of infectivity and pathogenicity against $G$. mellonella. As for, the infected G. mellonella revealed variable aspects on the cadavers post exposure (IJs), such as the muscles that suffered destruction in the fibrillate with some fragments and fat body tissues that showed high vacillations (Nouh and Hussein, 2014).

In conclusion, the mortality percentage of EPNs $(H$. bacteriophora and $S$. carpocapsae) increased gradually with the increase in exposure period and concentration of the tested insecticides. Dipel $2 \mathrm{x}$ and Radiant insecticides have slight toxicity to tested EPNs. However, Proclaim, Aphox and Coragen insecticides are harmful to test EPNs under laboratory conditions. As for, Proclaim treatment resulted to the highest mortality of studied EPNs. Overall, Atwa et al. (2009) found that the pathogenicity of treated $H$. bacteriophora by Proclaim ${ }^{\circledR}$ insecticide was negatively affected; this result was in line with those reported for Proclaim ${ }^{\mathbb{B}}$ insecticide (Koppenhöfer and Kaya, 1998). Future research is in dire need to explore and exploit the role of EPNs as biological control elements without influenced by synthetic pesticides in IPM programs.

\section{REFERENCES}

Atwa, A. A., A. F. B. El-Sabah and M. M. Gihad (2009). The effect of different biopesticides on the cabbage white butterfly, Pieris rapae (L.) in cauliflower fields. Alexandria Journal of Agricultural Research, 54(1): 147-153.

Bortoluzzi, L., L. F. A. Alves, V. S. Alves and N. Holz (2013). Entomopathogenic nematodes and their interaction with chemical insecticide aiming at the control of banana weevil borer, Cosmopolites Sordidus Germar (Coleoptera: Curculionidae). Arquivos do Instituto Biologico (Sao Paulo), 80(2): 183-192.

Glazer, I. and E. E. Lewis (2000). Bioassays for entomopathogenic nematodes. In: Navon, A. and Ascher, K.R.S. (eds) Bioassays of Entomopathogenic Microbes and Nematodes. CAB International, Wallingford, UK, pp. 229238. https://WWW.APC.gov.eg

Kaya, H. K., A. Alunmai, H. Y. Choo, T. M. De La, A. Fodor, S. Ganguly, S. Hazir, T. Lakatos, A. Pye, M. Wilson, S. Yamanaka, H. Yang and RU. Ehlers (2006). Status of entomopathogenic nematodes and their symbiotic bacteria from selected countries or regions of the world, 38: 134-155.

Kaya, H.K. and T. M. Burlando (1989). Development of Steinernema feltiae (Rhabditida: Steinernematidae) in diseased insect hosts. Journal of Invertebrate Pathology, 53: 164 168.

Kaya, H. K., A. M. Koppenhöfer and M. Johnson (1998). Natural enemies of entomopathogenic nematodes. Japanese Journal of Nematology, 28: 13-21.

Koppenhöfer, A. M. and H. K. Kaya (1998). Synergism of Imidacloprid and an entomopathogenic nematode: a novel approach to white grub (Coleoptera: Scarabaeidae) control in turf grass. Journal of Economic Entomology, 91(3):618-623.

Laznik, Z. and S. Trdan (2013). Testing the compatibility of different insecticides with entomopathogenic nematodes (Nematoda: Rhabditida) [Conference poster]. Zbornik Predavanj in Referatov, 11. Slovenskega Posvetovanja o Varstvu Rastlin Z Mednarodno Udeležbo (in okrogle mize o zmanjšanju tveganja zaradi rabe FFS $\mathrm{v}$ okviru projekta 
CropSustaIn), Bled, Slovenia, 5.-6. Marec, 2013 pp. 327-333.

Mahmoud, M. F. and M. A. M. Osman (2007). Relative toxicity of some bio-rational insecticides to second instar larvae and adults of onion thrips (Thrips tabaci Lind.) and their predator Orius albidipennis under laboratory and field conditions. Journal of Plant Protection Research, 47(4): 391-400.

Mahmoud, M. F. (2007). Combining the botanical insecticides NSK extract, Neem Azal T 5\%, Neemix $4.5 \%$ and the entomopathogenic nematode Steinernema feltiae Cross N 33 to control the peach fruit fly Bactrocera zonata (Saundres). Plant Protection Sci., 43(1): 19-25.

Mahmoud, M. F. (2016). Biology and use of entomopathogenic nematodes in insect pests biocontrol, a generic view. Cercetări Agronomiceîn Moldova, Vol. XLIX, No. 4 (168): 85-105.

Mahmoud, M. F., H.M. Mahfouz and K. M. Mohamed (2016). Compatibility of entomopathogenic nematodes with neonctinoid and azadirachtin insecticides for controlling the black cutworm, Agrotis ipsilon (Hufn.) in canola plants. International Journal of Research in Environmental Science, 2(1): 11-18.

Morales-Rodriguez, A. and D. C. Peck (2009). Synergies between biological and neonicotinoid insecticides for the curative control of the white grubs Amphimallon majale and Popillia japonica. Biological Control, 51(1): 169-180.

Nouh, G. M. and M. A. Hussein (2014). Virulence of Heterorhabditis bacteriophora (Rhabditida: Heterorhabditidae) produced in vitro against Galleria mellonella (Lepidoptera: Pyralidae). Research Journal of Pharmaceutical, Biological and Chemical Sciences, 5(3): 1385-1393.

Poinar, G. O. Jr, G. M. Thomas, G.M. and B. Lighthart (1990). Bioassay to determine the effect of commercial preparations of Bacillus thuringiensis on entomogenous Rhabditoid nematodes. Agriculture, Ecosystems and Environment, 30: 195-202.

SAS Institute Inc (2005). SAS/STAT User's guide. 6.12 edition. Cary, NC, SAS Institute Inc.

Sznyk-Basalyga, A. and A. Bednarek (2003).Effect of entomopathogenic nematodes and insecticides on Megaselia halterata mortality. Bulletin OILB/SROP, 26(1): 147-150.

Sznyk-Basalyga, A., A. Bednarek and M. Lewandowski (2002). Effect of simultaneous application of entomopathogenic nematodes and insecticide Trigard on flies Lycoriella solani (Diptera: Sciaridae) and Megaselia halterata (Diptera: Phoridae). Progress in Plant Protection, 42(2): 460-463.

White, G. F. (1927). A method for obtaining infective nematode larvae from cultures. Science 66: 302-303.

\section{تأثير بعض المبيدات على النيماتودا الممرضة للحشرات وتقييم مدى فاعليتها ضد دودة الشمع الكبرى تحت ظروف المعمل \\ دينا عطوة حسين"، منى أحمد حسين"، مصطفى أحمد الخواص'، عوض أحمد سرحان' ، ناصر سعيد مندور' ، محمود فرج محمود'

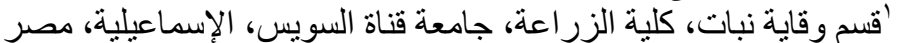

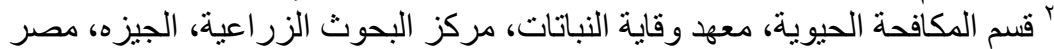

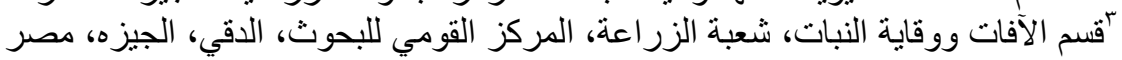

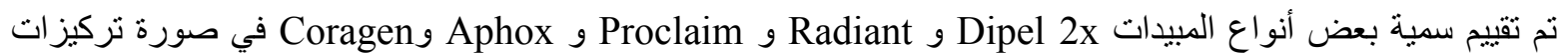

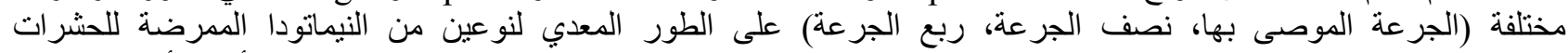
(Steinernema carpocapsae BA2 و والتي تعتبر من أهم الأعداء الحيوية

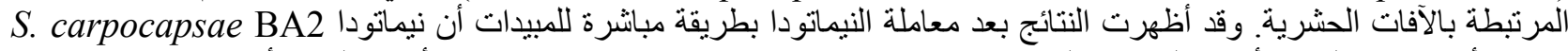

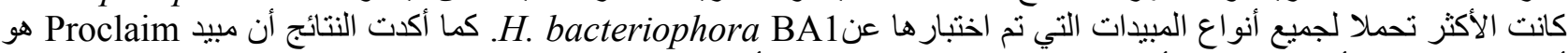

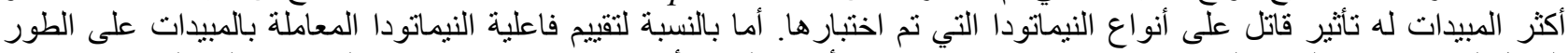

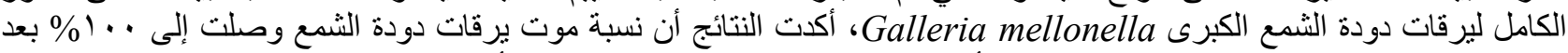

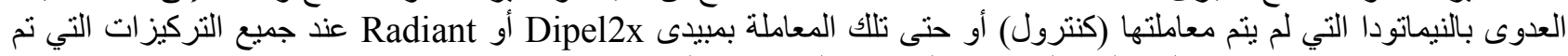

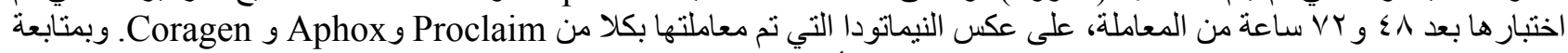

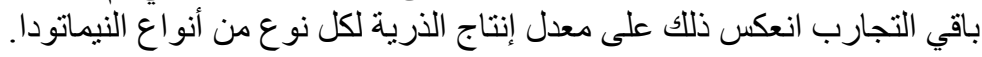

